\title{
Comparative evaluation of inverse scattering quantitative CT algorithms for sound velocity image reconstruction
}

\author{
Akira Yamada \\ Tokyo University of Agriculture and Technology, \\ 2-24-16, Nakamachi, Koganei, Tokyo, 184 Japan
}

(Received 6 February 1997)

\begin{abstract}
For the purpose of attaining higher accuracy of the $3 \mathrm{D}$ velocity quantitative CT image of the biological tissue based on the inverse scattering analytical method, introduction was made with such improved methods as of quasi-3D image reconstruction, correction of the $2 \mathrm{nd}$ order bias of the Rytov approximation based on the data collection on the aperture of a cylinder, together with its performance evaluation. As a result of the value test by means of the simulation for a sphere or cylindrical object, it is verified that a sound velocity quantitative CT image with by far higher accuracy and higher quality than the conventional one can be brought to realization under the condition of the sound velocity ratio and observation distance satisfying requirement for practicality.
\end{abstract}

Keywords: Ultrasonic CT, Diffraction tomography, Inverse scattering, Medical diagnosis, Quantitative image

PACS number: $40.80 . \mathrm{Vj}, 43.60 . \mathrm{Pt}$

\section{INTRODUCTION}

Ultrasonic CT (Computerized Tomography) is a method to reproduce physical parameter images of a object for sound speed or attenuation factor by solving an inverse scattering problem based on a mathematical model established between the measurement data and the acoustic physical quantities. Above all, the inverse scattering analytical images represented by the diffraction tomography method $^{1-3)}$ are the ones by a method to obtain an inverse scattering solution of a wave equation linearized under weak scattering approximation in accordance with computer processing, and the method can be realized by means of relatively-short-time calculation. A feature is pointed out, for example, with the fact that a stabilized solution can be obtained for the inputted noise or measurement errors. From this reason, high-resolution images can be accomplished to the extent of the wavelength order of the sound wave when the method in ques- tion is applied to the medical image diagnosis. In addition to the above, realization of the quantitative physical parameter images of the sound velocity or attenuation factor becomes possible, and the performance exceeding by far the ordinary B mode image can be expected. However as a major cause of the method in question not being put to practical use despite these excellent features, a problem is adduced in the fact that formation of an assumption of the linearity of the wave equation by means of Rytov approximation for too long an observation distance or a measurement objective with great sound velocity variation (i.e. with great scattering amplitude) becomes hard, resulting in exceeding deterioration in image performance.

In this study, the problem described above is to be confronted in accordance with a method executing backward propagation processing (backward propagation Rytov approximation) prior to the Rytov transformation of the observation data. As the second problem for the realization of the present 
method, transmission/receiving of sound waves and reconstruction calculation must be made 3dimentionally in order to deal with an inverse scattering problem of the actual 3D scattering phenomenon. However when such $3 \mathrm{D}$ reconstruction is intended to be brought to realization in a direct manner, an actual difficulty for the observation range to be restricted is constituted in addition to the trouble of increase in an amount of data collection or hardware scale (the observation on an intrinsic aperture on a sphere is a difficult one, and is restricted to the observation on an aperture on a plane or on a cylinder). To comply with this problem, exclusively the transmission/receiving data of the sound wave on an aperture of a cylinder is to be used in this study, and quasi-3D sound velocity parameter images are reproduced by conducting the spatial filter processing in a elevation direction and the $2 \mathrm{D}$ image reconstruction operation for the inside of the horizontal surface. In this paper, details of the quantitative image performance of the quasi-3D sound velocity image reconstruction method $^{4,5)}$ are examined from various points of view in practical use.

\section{PROBLEM SETTING}

As illustrated in Fig.1, there exists an inhomogeneous object with sound velocity $c(x, y, z)$, attenuation factor $\alpha(x, y, z)$ in the homogeneous medium with sound velocity $c_{0}$, attenuation factor 0 . Suppose that the original coordinate $(x, y, z)$ is rotated around $z$ axis by an angle $\theta$ to the rotated new axis $(X, Y, z)$. Let plane wave $\psi_{1}$ with the angular frequency $\omega$ be irradiated from backward of the $Y$ axis onto the object. That is,

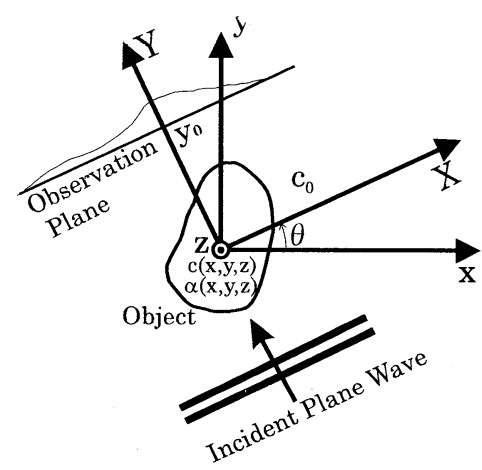

Fig. 1 Transmission/receiving of a sound wave.

$$
\psi_{\mathrm{i}}=\psi_{\mathrm{io}} \exp \left[j\left(k_{0} Y-\omega t\right)\right]
$$

where $k_{0}$ is wave number in the surrounding medium and $k_{0}=\omega / c_{0}$. On the individual points on the $(X, z)$ observation plane on a position of $Y=y_{0}$ in front of the object, let transmission wave $\psi_{\mathrm{M}}\left(X, z ; Y=y_{0}\right)$ be observed. Based on the measurement data $\psi_{\mathrm{M}}$ on the aperture surface around the $z$ axis of $\theta=0-360$ degree, let a problem allowing the object function $o(x, y, z)$ in the equation shown below to be quasi-3-dimensionally imagereproduced be considered.

$$
o(x, y, z)=\left\{k(x, y, z) / k_{0}\right\}^{2}-1
$$

where $k(x, y, z)$ is the wave number in the medium and is defined as shown below.

$$
k(x, y, z)=\omega / c(x, y, z)+\mathrm{j} \alpha(x, y, z)
$$

If the object function $o$ is obtained, then sound velocity distribution $c$ and attenuation factor distribution $\alpha$ can be obtained from the relation of Eqs. (2), (3).

\section{2D IMAGE RECONSTRUCTION}

\subsection{Problematic Points in the Conventional Method}

The diffraction tomography is the method based on the solution of the inverse scattering problem using the linearity assumption between the approximate transform of the observation data and the object function. For such a linearlization technique, Rytov approximation has been conventionally used to transform the original data $\psi_{\mathrm{M}}$ into the new data $\psi_{\mathrm{R}}$ which is given by

$$
\begin{aligned}
\psi_{\mathrm{R}} & =\psi_{i} \log \left(\psi_{\mathrm{M}} / \psi_{\mathrm{i}}\right) \\
& =\psi_{i} \log \left(\left|\psi_{\mathrm{M}} / \psi_{\mathrm{i}}\right|\right)+\mathrm{j}\left[\arg \left(\psi_{\mathrm{M}}\right)-\arg \left(\psi_{\mathrm{i}}\right)\right]
\end{aligned}
$$

Rytov approximation assumes that the total change of the phase and/or attenuation factor is the summation of them at each place along the propagation ray. Applying the Fourier diffraction slice theorem which relates the real part of $\psi_{\mathrm{R}}$ (relative log amplitude) with the attenuation factor $\alpha$ of the object and imaginary part of $\psi_{\mathrm{R}}$ (phase difference) with the inverse sound velocity $c^{-1}$, image of sound velocity $c$ and attenuation factor $\alpha$ can be finally obtained. The procedure of conventional 2D diffraction tomography method is summarized in Fig. 2(a).

A simulation evaluation test using the 2D recon- 


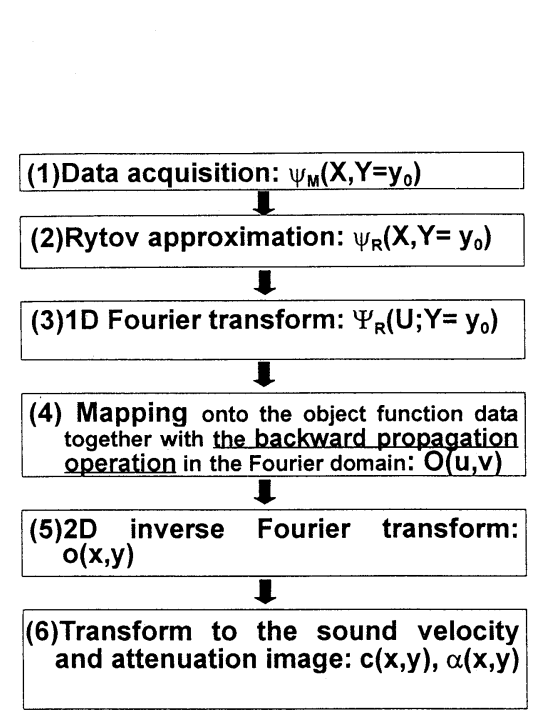

(a) Conventional method

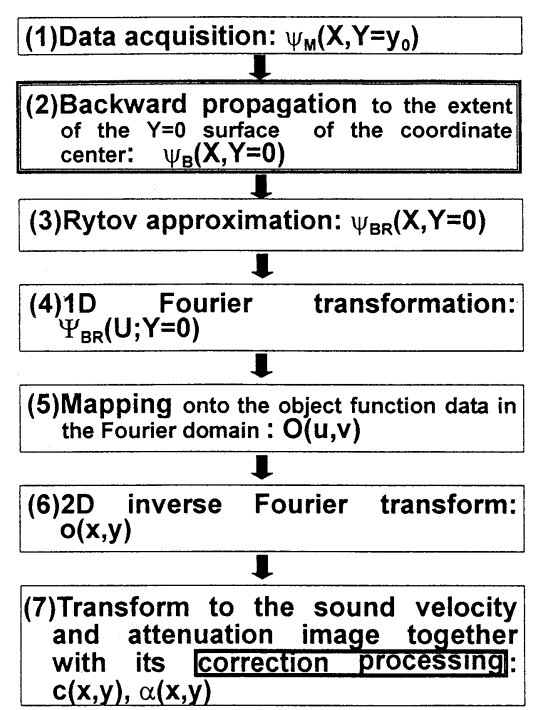

(b) Proposed method

Fig. 2 Procedure of 2D image reconstruction. (a) Conventional method, (b) Proposed method.
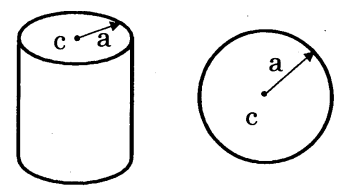

(a)Cylinder
(b)Sphere

Fig. 3 Configuration of test specimen.

struction method for a $2 \mathrm{D}$ cylindrical object is made in order to demonstrate the problem in question regardless of whether $3 \mathrm{D}$ handling is available or not. As an example, image calculation is made based on the simulation data of the transmission wave observed when a planar sound wave of $f_{0}=750$ $\mathrm{kHz}$ is irradiated onto the cylindrical specimen (Fig. $3(\mathrm{a})$ ) of radius $a=10 \mathrm{~mm}$, sound velocity $c=1.05 c_{0}$, attenuation factor $\alpha=0$ placed concentrically with coordinate origin. With the other cases, image calculation is made under the condition listed in Table 1 including the one since this time on. The reconstruction result of the sound velocity on the $x$ axis using the conventional method described above is sketched in Fig. 4 choosing the observation distance $y_{0}$ as a parameter, whereas how the average error $\varepsilon$ of the reconstruction sound velocity is changed as a function of the observation distance $y_{0}$ is depicted in Fig. 5. Here, $\varepsilon$ is the relative error of the mean value $\bar{c}_{\text {recon }}$ of the reconstruction sound velocity in the inside of the circle whose radius in

Table 1 Parameters used in simulation tests.

\begin{tabular}{|c|c|c|}
\hline \multicolumn{3}{|l|}{ Observation } \\
\hline Frequency & $: \omega / 2 \pi[\mathrm{kHz}]$ & 750 \\
\hline $\begin{array}{l}\text { Surrounding Sound } \\
\text { velocity }\end{array}$ & $: \quad c_{0}(\mathrm{~m} / \mathrm{s})$ & 1500 \\
\hline Observation distance & $: y_{0}[\mathrm{~mm}]$ & 50 (typ.) \\
\hline Sampling spacing & $: \Delta x[\mathrm{~mm}]$ & 1 \\
\hline Observation plane dim. & $: L_{x} \times L_{z}[\mathrm{~mm}]$ & $128 \times 128$ \\
\hline \multicolumn{3}{|l|}{ Reconstruction } \\
\hline & \\
\hline $\begin{array}{l}\text { Dimension of reconstructe } \\
\text { cube at a side }\end{array}$ & $: L_{\mathrm{p}}[\mathrm{mm}]$ & 90.5 \\
\hline \multicolumn{3}{|c|}{ Test specimen (cylinder or sphere) } \\
\hline Radius & $: a[\mathrm{~mm}]$ & $5,10,15$ \\
\hline Sound velocity & $c / c_{0}$ & $0.9-1.1$ \\
\hline Attenuation factor & $\alpha$ & 0 \\
\hline
\end{tabular}

the $(x, y)$ plane is $0.5 a$ including the one since this time on, and is evaluated by $\varepsilon=\left|\bar{c}_{\text {recon }}-c_{\text {exact }}\right| / c_{0}$. Judging from the result of these, it is explained that the image is considerably deteriorated as the observation distance $y_{0}$ progresses. The result shows that error is generated accompanied with the backward propagation operation executed after the Rytov transformation, and none of the good results are to be obtained except that measurement is made in the immediate vicinity of the object. On the other hand, the reconstructed results of the average sound velocity value $\bar{c}_{\text {recon }}$ in the cylinder specimen in relation to the settled sound velocity value $c_{\text {exact }}$ is 


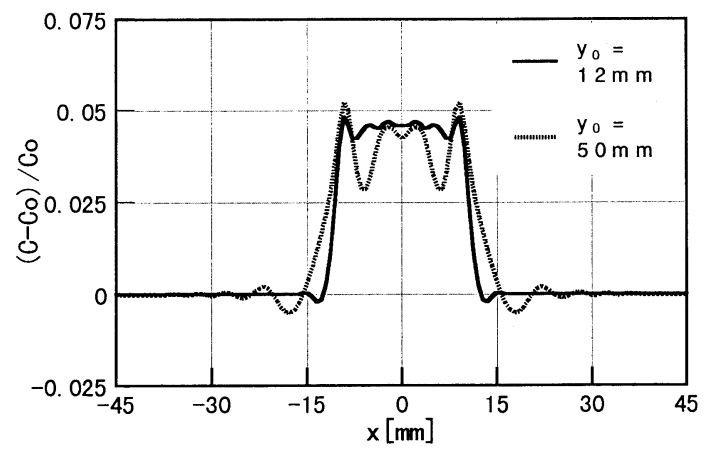

Fig. 4 Sound velocity reconstruction image of the cylindrical object using the conventional method.

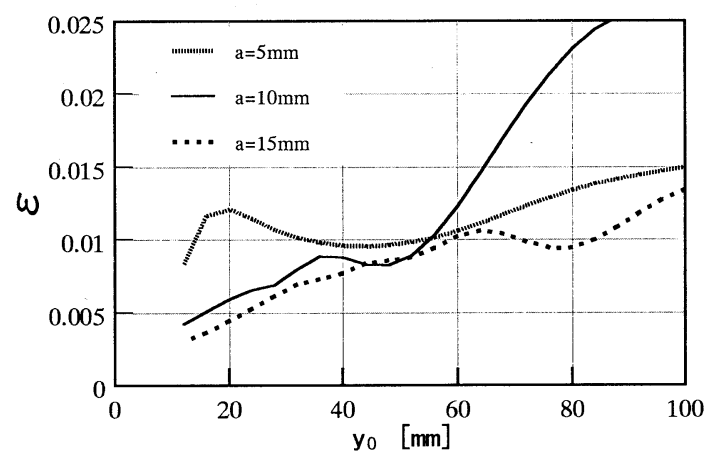

Fig. 5 Relation between the observation distance $y_{0}$ and the average error $\varepsilon$ of the reconstruction sound velocity using the conventional method.

shown in Fig. 6 which is conducted under the condition that $y_{0}=50 \mathrm{~mm}$. Because of the violation of the weak scattering approximation, the image is exceedingly deteriorated when the sound velocity difference $\Delta c_{\text {exact }} / c_{0}\left(=\left(c_{\text {exact }}-c_{0}\right) / c_{0}\right)$ exceeds the neighborhood of $6,7 \%$, resulting in failure of quantitative accuracy.

\subsection{Backward Propagation Rytov Approxi- mation $^{4,5)}$}

Above all, the deterioration of the image in case of too long an observation distance $y_{0}$ or in case of large sound velocity difference $\Delta c / c_{0}\left(=\left(c-c_{0}\right) / c_{0}\right)$ was pointed out in the conventional diffraction tomography. The cause of these types of trouble is originated from the fact that the conventional backward propagation operations executed on the Rytov transform data is failed. To resolve the problem, a method to execute the backward propagation

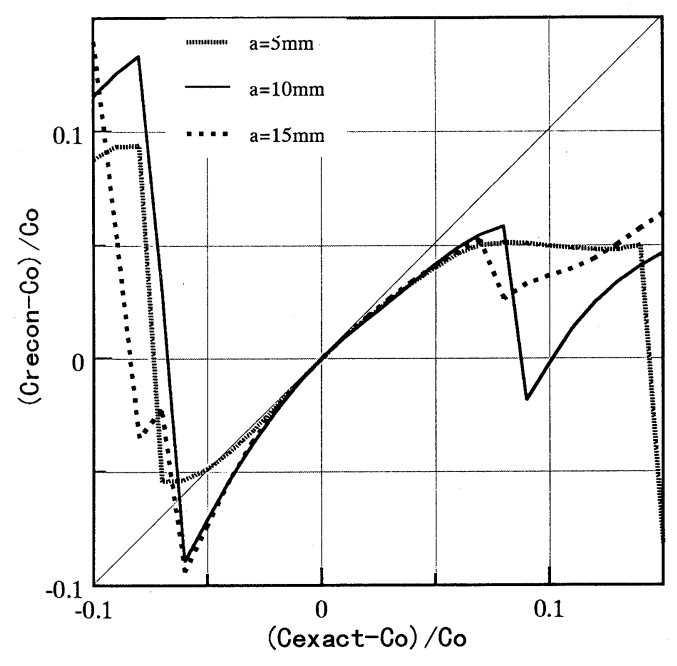

Fig. 6 Relation between the settled value of the sound velocity $c_{\text {exact }}$ of the cylindrical object and its reconstructed average value $\bar{c}_{\text {recon }}$ using the conventional method.

processing before Rytov transformation is presented in accordance with the procedure described in Fig. 2(b). Here the procedures surrounded by the thick rectangular frame indicate the ones being different from the conventional method (Bias correction procedure in (7) will be discussed in later section 4.2). With the use of the technique, the errors accompanied with the conventional backward propagation operation can be avoided.

To verify the effectiveness of the present method, let the sound velocity ratio of the cylindrical object be selected as $c=1.05 c_{0}, \alpha=0$, the radius as $a=10$ $\mathrm{mm}$. Here, the center of the cylinder is considered in coincidence with the coordinate origin, by which the backward propagation Rytov approximation might most effectively be in action. First of all, the result of the calculation of the complex scattering amplitude $\psi_{\mathrm{M}}\left(X, Y=y_{0}\right)$ observed on a position of $y_{0}=50 \mathrm{~mm}$ is illustrated in Fig.7. Next, the data was backward propagated to the extent of the $Y=$ 0 surface from a observation plane of $Y=50 \mathrm{~mm}$, and the Rytov transformation was applied. The result $\psi_{\mathrm{BR}}(X, Y=0)$ is illustrated in Fig.8 with the solid line. It can be seen that the real number part of $\psi_{\mathrm{BR}}\left(\log\right.$. amplitude of $\left.\psi_{\mathrm{B}}\left(=\log \left|\psi_{\mathrm{B}} / \psi_{\mathrm{i}}\right|\right)\right)$ takes a value as close as to zero, and the imaginary number part of $\psi_{\mathrm{BR}}$ (phase of $\psi_{\mathrm{B}}\left(=\angle\left(\psi_{\mathrm{B}} / \psi_{\mathrm{i}}\right)\right.$ ) exhibits a value corresponding to the whole amount of the inverse sound velocity change in the inside of the 


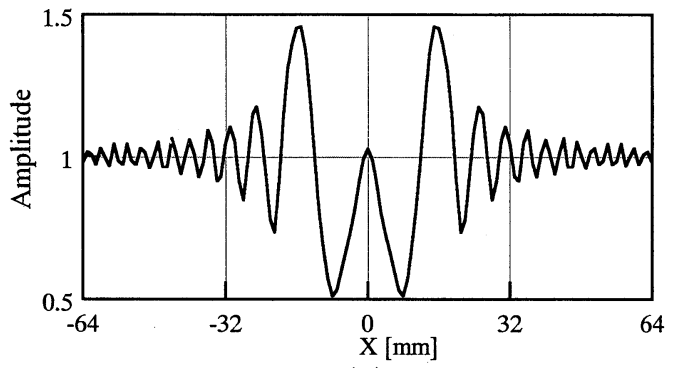

(a)

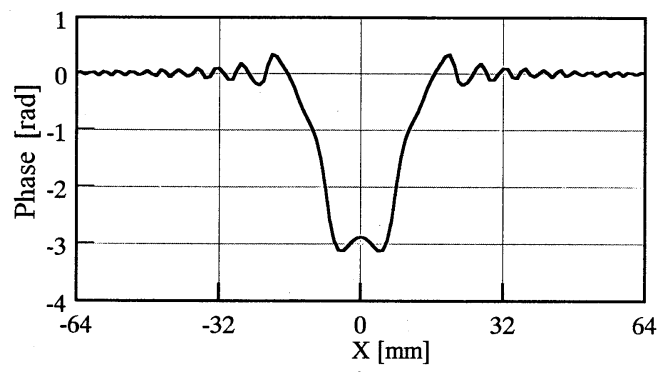

(b)

Fig. 7 Complex amplitude of the scattered field $\psi_{\mathrm{M}}\left(X, Y=y_{0}\right)$ from the cylinder specimen on an observation line. (a) Amplitude characteristics, (b) Phase characteristics.

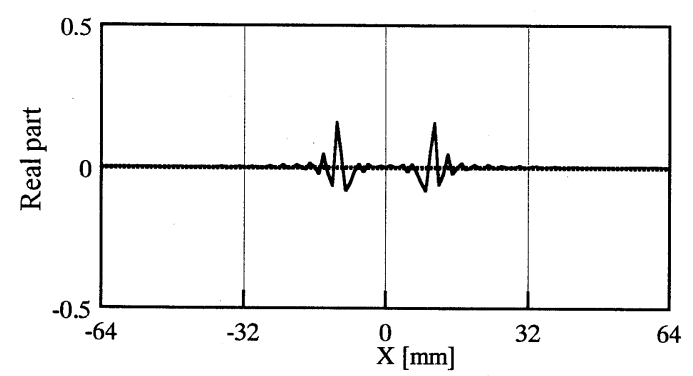

(a)

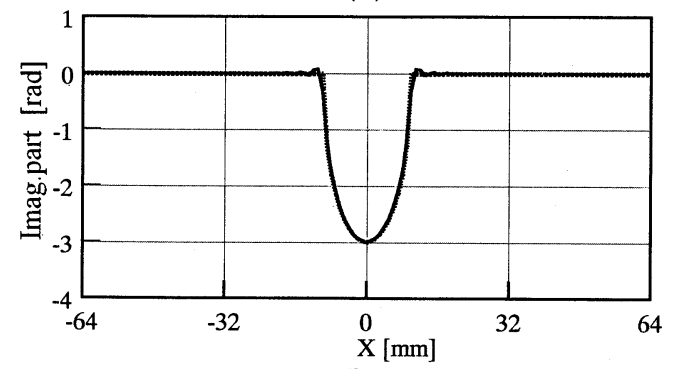

(b)

Fig. 8 Backward propagation Rytov transformation data $\psi_{\mathrm{BR}}(X, Y=0),-$ : calculated value, $\cdots$ : ideal value. (a) Real number part (log. amplitude characteristics), (b) Imaginary number part (phase characteristics).

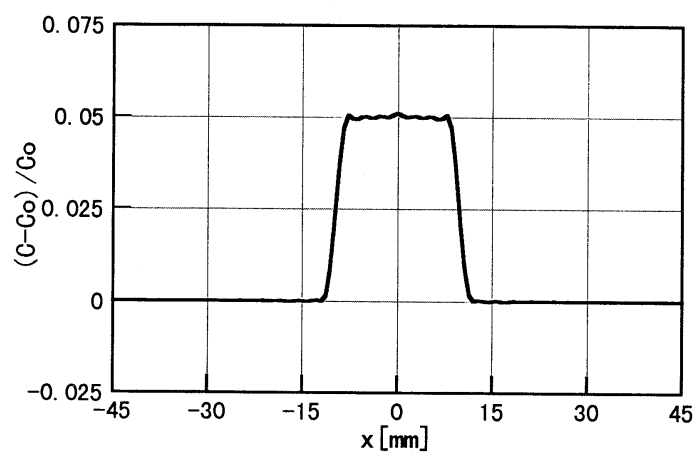

(a)

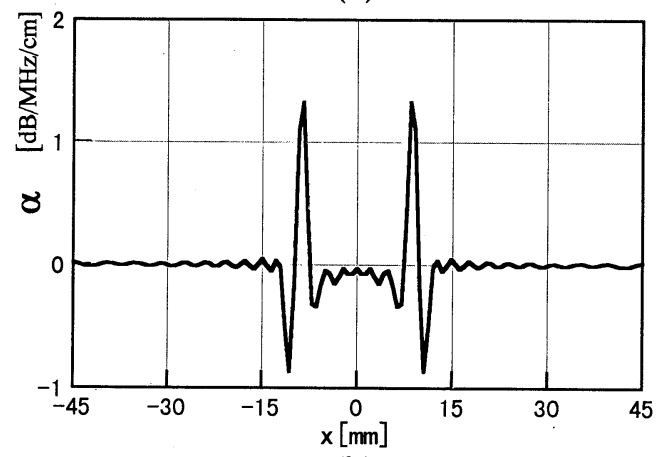

(b)

Fig. 9 Reconstruction result of the cylindrical object ( $a=10 \mathrm{~mm}, c=1.05 c_{0}, \alpha=0.0$ ) based on the backward propagation Rytov transformation. (a) Sound velocity image, (b) Attenuation factor image.

cylindrical object along a sound propagation path. The result thus obtained is close enough to coincide with the result expected to be brought about when the sound wave is ideally propagated to the receiver with a straight ray path in the object (The ideal values are indicated with the dotted lines).

Under the same condition as in Fig. 4, the final reconstruction result of the sound velocity image based on the present backward Rytov approximation is illustrated in Fig. 9(a). It can be seen that image with higher accuracy and higher quality is obtained compared with the result of the conventional method in Fig. 4. On the other hand, a result of the average error $\varepsilon$ of the reconstructed sound velocity as a function of the observation distance $y_{0}$ is illustrated in Fig. 10. From this it is confirmed that a highly accurate sound velocity image fundamentally independent of the observation distance is obtained. In particular, increase of 1 or 2 digit accuracy is made compared with the 


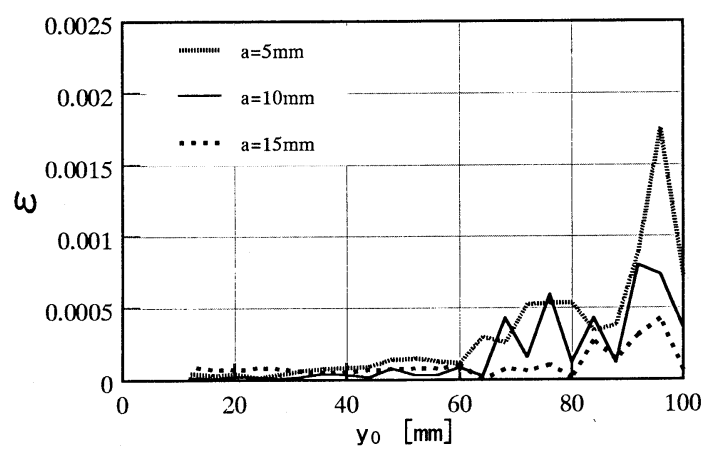

Fig. 10 Relation between the observation distance $y_{0}$ of the cylindrical object and the average error $\varepsilon$ of the reconstructed sound velocity based on the backward Rytov data.

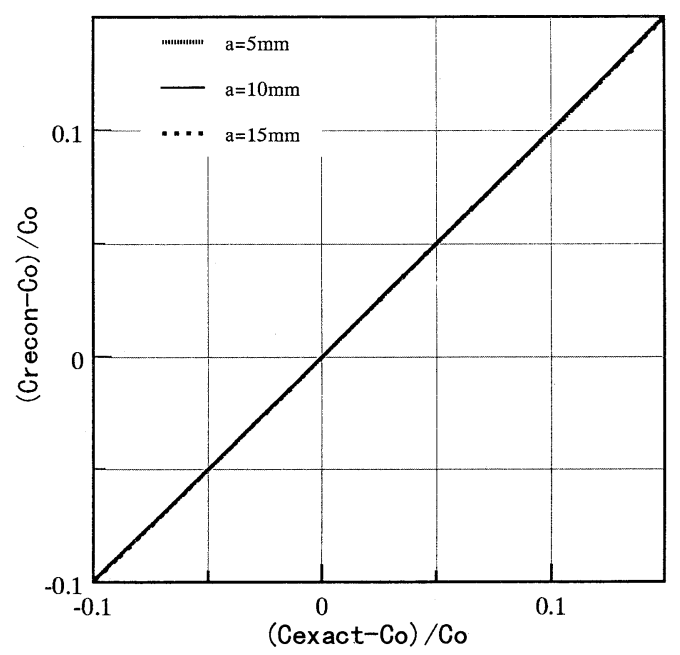

Fig. 11 Relation between the settled value $c_{\text {exact }}$ of the cylinder sound velocity and its average reconstructed value $\bar{c}_{\text {recon }}$ based on the backward propagation Rytov transformation.

result of the conventional method in Fig. 5. Meanwhile under the condition that $y_{0}=50 \mathrm{~mm}$, relation between the reconstructed average sound velocity value $\bar{c}_{\text {recon }}$ in the cylinder specimen and the settled value $c_{\text {exact }}$ is illustrated in Fig. 11. From the above, it is explained that the result with good quantitative accuracy is obtained to the extent of the sound velocity difference $\Delta c_{\text {exact }} / c_{0}$ as high as $10 \%$. Also it is explained that the sound velocity range where weak scattering approximation is valid is broadened compared with the case in Fig. 6 by means of the present backward propagation Rytov approximation.
By the way, since the present approximation method is intrinsically not the one to intend the direct mitigation of the weak scattering condition, the fact in the above observation appears to be an unexpected result. With respect to the reason for the above, the following can be considered. That is to say, although the real part (log. amplitude data) of the backward propagation Rytov data in Fig. 8 (a) is intrinsically to become zero, but an error is produced in the vicinity of the boundary of the cylinder owing to the influence of the Rytov approximation. Contrarily to the above, characteristics close enough to be ideal values are obtained with the imaginary part (phase data) in Fig. 8(b). In this connection, the attenuation factor images (the images of mainly the imaginary part) is shown in Fig. 9(b) together with the sound velocity images (the images of mainly the real part) in Fig. 9(a). Although the attenuation factor image might intrinsically become zero (attenuation of a cylinder specimen was determined to be zero), it is noticed that a great error is produced on the cylinder boundary owing to the error caused by the amplitude characteristics in Fig. 8(a). From these results, it can be considered that an approximate error produced by the backward propagation Rytov approximation processing mainly appears on the imaginary part (attenuation image) of the reconstruction image, and never appears so strongly on the real part (sound velocity image). That is to say, almost all of the weak scattering approximation errors are insulated on the imaginary part, and the image is believed to be reproduced with excellent accuracy even in case that the sound velocity difference is great.

\section{QUASI-3D IMAGE RECONSTRUCTION}

\subsection{Spatial Filter Processing ${ }^{4,5)}$ in a $z$ Direction}

A $2 \mathrm{D}$ object which is uniform in a $z$ direction is considered as a premise with the $2 \mathrm{D}$ image reconstruction method referred to above. However, for the application of the present method to the actual 3D object, 3D treatment is necessary compatible with the data from the restricted observation range. To this end, a quasi-3D multi-layered sound velocity image reconstruction method incorporating spatial filter processing in a $z$ direction is considered based on the transmission/receiving data of the sound wave on an aperture on the cylinder around 


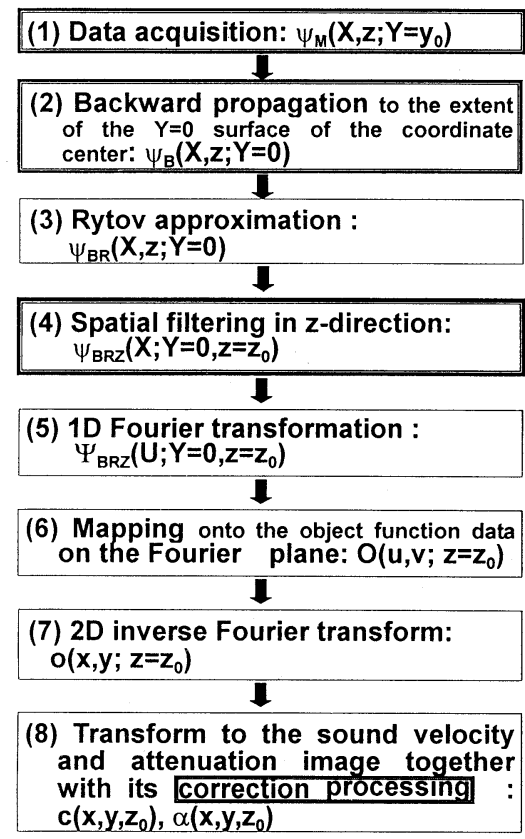

Fig. 12 Procedure of quasi-3D image reconstruction.

single rotational $z$ axis. To be concrete, the quasi$3 \mathrm{D}$ sound velocity images are reconstructed with the procedure as shown in Fig. 12. That is, after sound wave data are acquired over an $(X, z)$ 2D observation plane, then data are backward propagated and Rytov transformed as described before. Hereafter, spatial filtering processing in a $z$-direction is introduced. Finally, quasi-3D sound velocity parameter images for the inside of the horizontal $(x$, $y)$ plane are reproduced from the procedures similar to the conventional 2D image reconstruction operation.

As a $3 \mathrm{D}$ object, a spherical specimen as shown in Fig. 3(b) with a radius $a=10 \mathrm{~mm}$, sound velocity ratio $c=1.05 c_{0}$, attenuation factor $\alpha=0$ is placed on a position concentrically with the coordinate origin. Let it be imagined that the transmission scattered wave in case of its planar wave irradiation is observed on an observation surface of $y_{0}=50 \mathrm{~mm}$. The 3D sound velocity images on both the $x$ and $z$ axes that are reconstructed with the simulation data by applying the present method are respectively sketched in Figs. 13 (a) and (b) in solid lines. The sparse broken lines in the figures indicate the results obtained by applying the conventional 2D image reconstruction method in 3.1 using the $1 \mathrm{D}$ observa-

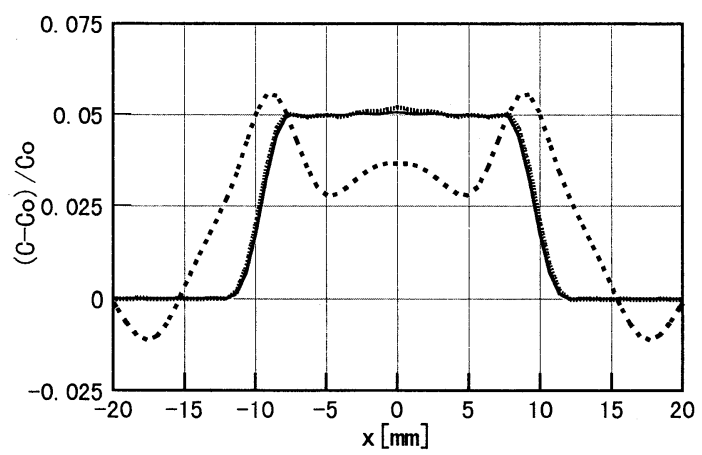

(a)

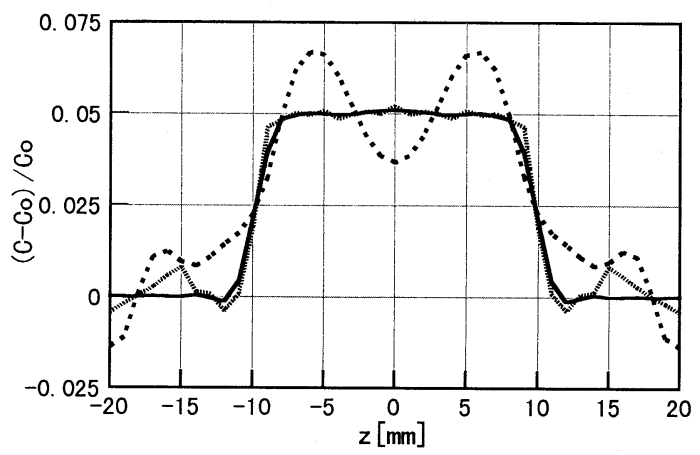

(b)

Fig. 13 Quasi-3D sound velocity reconstruction image of the spherical object, - : proposed method, $\cdots$ : conventional method, $\cdots: 2$-D conventional method. (a) Image on a $x$ axis, (b) Image on a $z$ axis.

tion data $\psi_{\mathrm{M}}\left(X ; Y=y_{0}\right)$. The same as seen in the result shown in 3 , the image is considerably deteriorated in comparison with the result expressed by the solid lines. Thus the effect of the backward Rytov approximation in this proposal can be confirmed. On the other hand, the dense broken line indicates the result in case that the spatial filter in (4) is omitted in the method of this proposal referred to above. Although difference from the solid line in the $\mathrm{x}$ direction in Fig. 13(a) is the slightest, great error appears in the neighborhood of the cylinder boundary with the result in the $z$ direction in Fig. 13(b). Thus the effect of the filter processing in question can be confirmed.

\subsection{Correction of Second Order Bias ${ }^{6)}$}

From the observation for the case that $\omega$ is dealt with as infinite, it is perceived that the reconstructed sound velocity based on the 1st order Rytov approximation yields deviation in a sense of the 2 nd order 
approximation. From this, 2nd order bias of the reconstructed sound velocity $c_{\text {recon }}$ can be corrected to the exact value $c_{\text {exact }}$ in accordance with the equation shown below.

$$
\Delta c_{\text {recon }} / c_{0}=\Delta c_{\text {exact }} / c_{0}+\left(\Delta c_{\text {exact }} / c_{0}\right)^{2},
$$

where $\Delta c_{\text {exact }}\left(=\left(c_{\text {exact }}-c_{0}\right) / c_{0}\right)$ and $\Delta c_{\text {recon }}(=$ $\left.\left(c_{\text {reaco }}-c_{0}\right) / c_{0}\right)$ are each sound velocity differences with respect to $c_{0}$.

Note that the present technique described above is valid for arbitrary object regardless of the shape of the object or dimension of the reconstruction. To verify the effectiveness of the correction method in question, evaluation error for the reconstructed value $c_{\text {recon }}$ before making the correction is shown in Fig. 14(a). Here the calculation condition is the same as in 4.1 referred to above with the case that the radius is selected as $a=5,10,15 \mathrm{~mm}$. The error $\varepsilon$ coincides with the square characteristics of $\Delta c_{\text {exact }} / \mathrm{c}_{0}$ regardless of how the object dimensions are. On the other hand, the error $\varepsilon$ for the corrected value

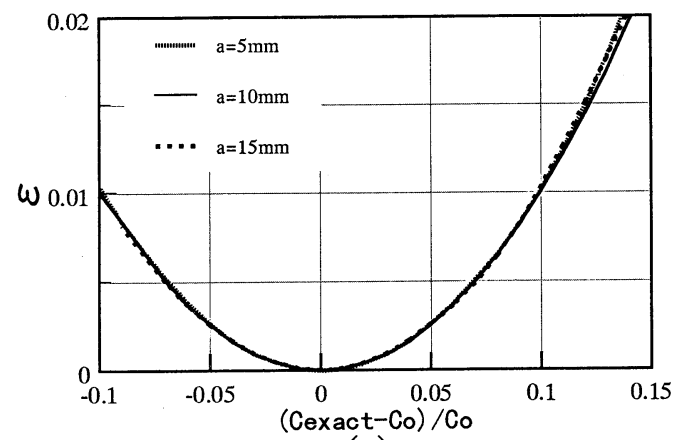

(a)

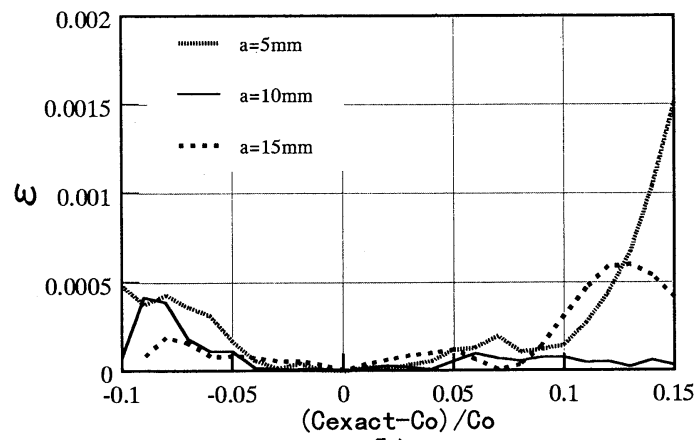

(b)

Fig. 14 Relation between settled value $c_{\text {exact }}$ of the sphere sound velocity and its evaluation error $\varepsilon$ (average error), (a) Before correction, (b) After correction.

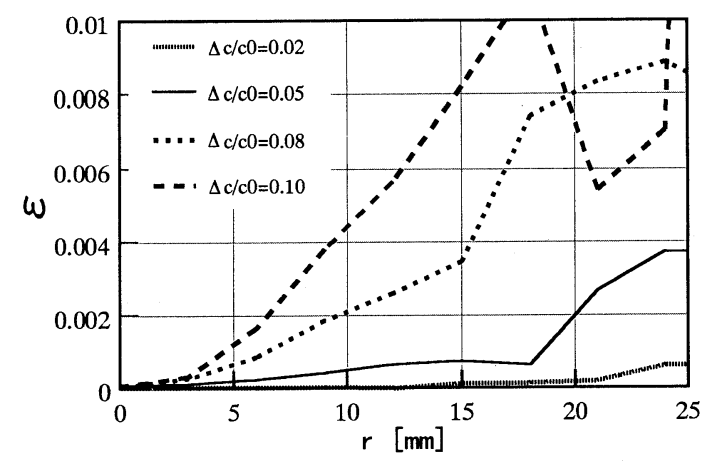

Fig. 15 Relation between the sphere center distance $r$ and the average error $\varepsilon$ of the reconstruction sound velocity.

obtained based on Eq.(5) is illustrated in Fig. 14(b). It is ensured that deviation is almost perfectly removed, and increase of more than 1-digit accuracy is successfully made throughout a broad sound velocity difference range of around $10 \%$.

4.3 Relation between the Object Position and the Image Error

With the backward propagation Rytov approximation shown in 3.2 , it is to be understood as the premise condition in theory that the scattering object center is extremely close to the coordinate origin. Therefore as the object recedes from the center, the accuracy is made deteriorated. To argue this matter, the center of the spherical object with radius $a=10 \mathrm{~mm}$ is settled on the position of $\left(x_{0}, y_{0}\right.$; $\left.z_{0}=0\right)$ separated from the origin on the $\left(x, y ; z_{0}=0\right)$ plane at a distance $r=\sqrt{x_{0}^{2}+y_{0}^{2}}$. A relation between the average error $\varepsilon$ of the reconstructed sound velocity image on the plane of $(x, y ; z=0)$ and the distance $r$ is shown in Fig. 15 changing the sound velocity of the sphere as a parameter. In case that the sound velocity difference $\Delta c / c_{0}$ is small, the quantitative accuracy is relatively good even if $r$ is great. However it is revealed that as the sound velocity difference $\Delta c / c_{0}$ is increased to the extent more than approximately $5 \%$, it becomes gradually impossible for the influence of the distance not to be ignored.

\subsection{Stability for the Measurement Error}

When application to real environment is considered, liability to error in measurement by the causes such as external mixed noises, deviation of the irra- 


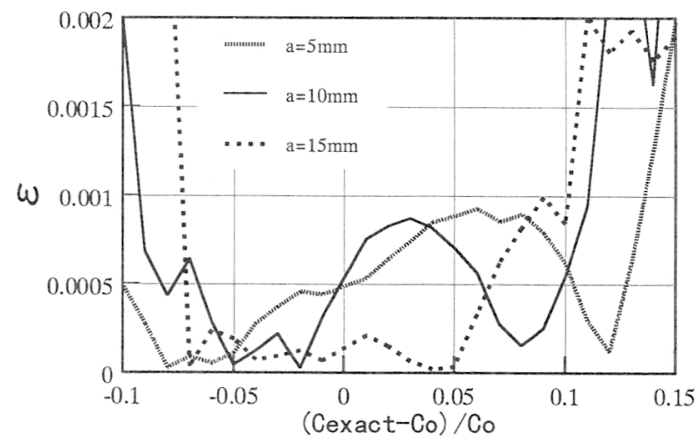

Fig. 16 Relation between the settled value $c_{\text {exact }}$ of the sphere sound velocity and its evaluation error $\varepsilon$ (average error) in case that the observation noise $(\mathrm{S} / \mathrm{N}$ ratio $20 \mathrm{~dB})$ is added.

diation wave from the ideal planar field, can never be avoided. Such being the case, stabilization in the accuracy of reconstructed image is very important from a point of view of practicality even if a considerable degree of great observation error is included in the inputted data. To achieve such conduct, a relation between the settled value $c_{\text {exact }}$ of the sound velocity of the spherical object and the average error $\varepsilon$ is obtained under the computational condition the same as the one in Fig. 14, which is previously referred to for each of the amplitude of the scattering inputted data and the phase to which the random noise simulated with the measurement noise having the $\mathrm{S} / \mathrm{N}$ ratio equivalent to $20 \mathrm{~dB}$ is added. The result thus obtained is shown in Fig. 16. Since the random noise is in assumption despite the fact that a relatively great error is supposed, deterioration of the quantitative accuracy is not so large with respect to the average value. Thus it is conceived that a stabilized solution is obtained for the measurement noise.

\section{CONCLUSIONS}

On the assumption that the argument is made in the event of the object center being located in the vicinity of the origin of the coordinate, it is verified that the effect of the backward propagation approximation is considerably effective. It is also verified that sound velocity images can be reproduced to the extent of the accuracy as high as none of problems being caused practically even when the sound velocity of the object also varied in a range of approximately $10 \%$. Also as a result of the investigation concerning the effectiveness of the quasi-3D image reconstruction method based on the observation data on the aperture on the cylinder, correction processing to eliminate the 2 nd order bias of the Rytov approximation, stability for the measurement noise, etc., it is imagined that the problems in practicality now will successfully have been solved. In future a countermeasure not to allow the image deterioration for the long separation distance between the object center and the coordinate origin is to be made.

\section{REFERENCES}

1) K. Mueller, M. Kaveh, and G. Wade, "Reconstructive tomography and applications to ultrasonics," Proc. IEEE 67, 567-587 (1979).

2) M. Kaveh and R. D. Iverson, "A new approach to acoustic tomography using diffraction techniques," Acoust. Imaging 8, 615-628 (1980).

3) A. Yamada and K. Kurahashi, "Experimental image quality estimation of ultrasound diffraction tomography,” Jpn. J. Appl. Phys. 32, 2507-2509 (1993).

4) A. Yamada, "Quasi 3D quantitative computerized tomography for reconstructing sound velocity of a weakly scattering object," Jpn. J. Appl. Phys. 35, 163 -166 (1996).

5) A. Yamada, "Inverse scattering quantitative computerized tomography for the sound velocity measurement of biological tissues," J. Acoust. Soc. Jpn. (J) 52, 716-721 (1966) (in Japanese).

6) A. Yamada, "High precision quantitative reconstruction of inverse scattering sound velocity image by the corrected Rytov approximation method," 17 th Symp. Ultrason. Electron. OK4, 127-128 (Oct. 1996/Yonezawa) (in Japanese).

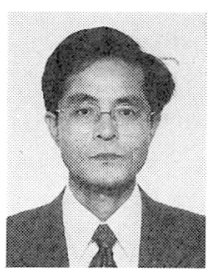

Akira Yamada received his B. S., M. S., and Ph. D. degrees in electrical communication engineering in 1976, 1978 and 1982, respectively, from Tohoku University, Sendai, Japan. He was Assistant Professor at Toyohashi University of Technology, Toyohashi, Japan, from April 1982 to January 1989, and then served as an Associate Professor in the Department of Electrical Engineering at Tokyo University of Agriculture and Technology, Tokyo, Japan, from February 1989 to March 1995. Since April 1995 he has been with the graduate school of Bio-Applications \& Systems Engineering at Tokyo University of Agriculture \& Technology. His research interests include signal processing in applied ultrasonic measurement, ultrasonic computerized tomography and ultrasonic inverse scattering problem. Dr. Yamada is a member of the Acoustical Society of Japan, the Institute of Electronics Information and Communication Engineers of Japan, and the Institute of Electrical and Electronics Engineers. 